\title{
Nanotribology of Poly(dimethylsiloxane) Melt Confined between Hydrophobic Surfaces
}

\author{
Shinji Yamada ${ }^{*}$ \\ Analytical Research Center, Kao Corporation \\ 2606 Akabane, Ichikaimachi, Haga, Tochigi 321-3497, Japan \\ *Corresponding author: yamada.s@kao.co.jp
}

( Manuscript received 24 August 2006; accepted 10 October 2006; published 31 October 2006 )

\begin{abstract}
Friction measurements were carried out for a poly(dimethylsiloxane) (PDMS) melt ( $M_{\mathrm{w}} \approx 80000$ ) confined between hydrophobic surfaces using the surface forces apparatus. The PDMS films were prepared by two different procedures: i) compression of a droplet into a hard-wall state (compressed system); ii) adhesive contact of two thin films cast on each substrate from solution (cast system). The dynamic thicknesses were 1.4 $\mathrm{nm}$ for the compressed system and $2.0 \mathrm{~nm}$ for the cast system. Despite the large thickness, the friction of the cast system was larger than that of the compressed system. Large thicknesses generally give low friction; the unusual result suggests that the confined structures may be different between the two systems. The PDMS molecules in both systems lay parallel to surfaces, but the extent of ordering could be much higher for the compressed system. The compressed film has a layer structure and slipping mainly occurs between the layers, resulting in the low friction. On the contrary, the cast system should have a disordered structure; molecules may interdigitate to each other and possibly form bridges across the sliding surfaces, which could induce large friction. The effect of film the preparation procedures on molecular ordering is discussed.
\end{abstract}

Keywords: surface forces apparatus, nanotribology, confinement, poly(dimethylsiloxane), molecular layering, squeeze flow

\section{Introduction}

The structures and dynamics of thin polymer melt films confined between two surfaces are of importance in many academic research areas as well as for several practical applications such as lubrication and polymer processing. Due to the ability to measure the dynamic thickness and shear force of an intervening film accurately during sliding, the surface forces apparatus (SFA) technique has been employed to study the dynamic behavior ${ }^{1-4)}$. One of the characteristic observations of confined polymer melts compared with simple liquid lubricants is relatively large dynamic thicknesses ${ }^{5,6}$. When confined between two surfaces by normal compression, polymer melts often produce nonequilibrium repulsions such as steric forces and/or viscosity forces, which tend to prevent the thickness decrease $^{7)}$. If the polymer melts have large chain entanglement effects, the nonequilibrium interactions are sometimes large enough for the films to stabilize against compression at thicknesses roughly comparable to the size of the random coil dimensions.

In a previous study ${ }^{8,9)}$, we investigated the structures and dynamics of a confined poly(dimethylsiloxane)
(PDMS) melt using the SFA. The molecular weight of the PDMS melt was about 80000 , which is large enough to have chain entanglement effect (an entanglement limit of PDMS is 12000) ${ }^{10}$ ). However, the results showed that the dynamic thicknesses equal to two, three or four molecular layers, which are an order of magnitude thinner than the typical size of the random coil conformation. Measured friction properties also suggested that the extent of ordering of PDMS molecules in the films is exceptionally high; shear is mainly accomplished by the slipping between the layered planes. However, the mechanism underlying this exceptionally high molecular ordering (layering) of the polymer chains is not clear.

In this paper, we describe the tribological experiment of the thin PDMS films, prepared by two different procedures, sheared between two hydrophobic surfaces. The nanometer-thick films of PDMS melt were cast on hydrophobic substrates from solution, and the two cast films were made in adhesive contact. The friction of the film was measured using the SFA, and the results were compared with those of a hard-wall PDMS film prepared by the compression of a bulk droplet. The effect of film preparation procedures on the molecular 
ordering (layering) is discussed, which gives us a new insight to obtain highly-ordered layer structures for long chain polymer melts in confinement.

\section{Experimental Methods}

The poly(dimethylsiloxane) (PDMS) melt investigated is a commercial silicone oil obtained from Toray Dow Corning Silicone Co., Japan and was used as received ${ }^{8)}$. The weight-average molecular weight $M_{\mathrm{w}}$ is about 80000 and the estimated polydispersity of the sample is 1.4. The size of the random coil conformation is estimated to be about $10 \mathrm{~nm}^{9)}$, and the width of the PDMS chain is about $0.7 \mathrm{~nm}^{11)}$.

The hydrophobic surface used in this study was a self-assembled monolayer of double-chained surfactant DDAB (Didodecyldimethylammonium bromide, ACROS Organics, USA) on cleaved mica ${ }^{8,12)}$. The molecular area was about $0.50 \mathrm{~nm}^{2}$ per molecule, which corresponds to an almost close-packed monolayer for this surfactant. The surface energy was $25 \mathrm{~mJ} / \mathrm{m}^{2}$ for the monolayer ${ }^{8,12}$.

\section{a) Compressed system}
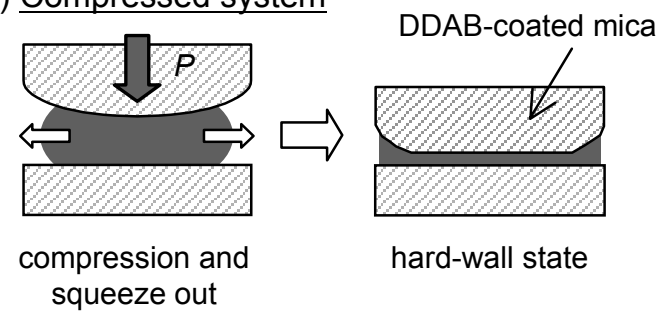

hard-wall state

b) Cast system

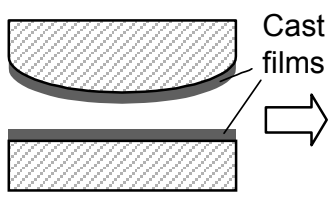

approach in air

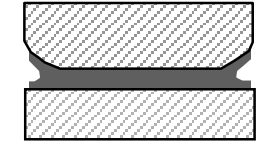

adhesive contact

Fig.1 Two different procedures to prepare molecularly thin PDMS films between hydrophobic surfaces.

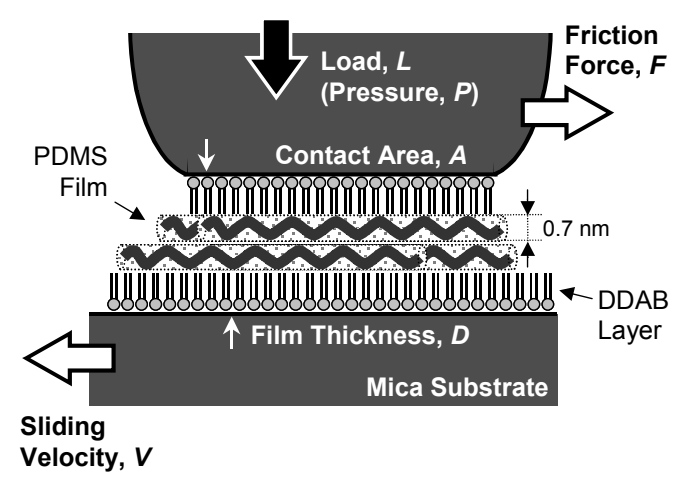

Fig.2 Schematic drawing of the contact region in the SFA friction measurements.
The compressed PDMS film was obtained by the following procedure. The two DDAB-coated mica surfaces were positioned in a crossed cylinder configuration in the measuring chamber of a SFA3 (SurForce Corp., USA) ${ }^{13)}$. The droplet of PDMS melt (vol. $\sim 0.1 \mathrm{ml}$ ) was put on the DDAB-coated surface using a thin syringe needle and then the chamber was purged with dry nitrogen gas for $12 \mathrm{~h}$. Some $\mathrm{P}_{2} \mathrm{O}_{5}$ was also placed inside the sealed chamber to keep the internal atmosphere completely dry at all times. The two surfaces were approached stepwise by decreasing surface distance (applying an normal pressure $P$ ). The interface was allowed to equilibrate at each step until the surface distance (PDMS thickness) became stable. Finally, the surfaces become flat because of the elastic deformation of the glue layer under each mica substrate as shown in Figure 1a. A hard-wall film was attained at $P=3.0 \mathrm{MPa}$. Before applying sliding, the hard-wall film was left to equilibrate for $1 \mathrm{~h}$.

The cast PDMS system was prepared as follows (Figure 1b). The PDMS was dissolved in hexane at concentration $0.03 \mathrm{mg} / \mathrm{mL}$. The solution (vol. $10 \mu \mathrm{L}$ ) was dropped and spread onto the DDAB-coated mica sheet (about $10 \times 10 \mathrm{~mm}^{2}$ ) and left for solvent evaporation. $^{\text {a }}$ The estimated thickness of the PDMS film was about $3 \sim 5 \mathrm{~nm}$, and the RMS roughness was less than $0.1 \mathrm{~nm}$ (obtained over an area of $3 \times 3 \mu \mathrm{m}$ using atomic force microscope). The two PDMS films cast on mica were installed into the SFA chamber and then the chamber was purged with dry nitrogen gas for $12 \mathrm{~h}$ and some $\mathrm{P}_{2} \mathrm{O}_{5}$ was also placed. When the two PDMS surfaces approached slowly, they jumped into adhesive contact at the surface distance about $20 \mathrm{~nm}$. The contact thickness decreased gradually and reached equilibrium in $1.5 \sim 2 \mathrm{~h}$. Friction measurements were made at the adhesive contact condition $(P=0)$.

The SFA was equipped with a bimorph slider that generates lateral motions at constant velocity $V(0.001$ to $0.2 \mu \mathrm{m} / \mathrm{s}$ ) and resulting friction force $F$ was measured by means of a friction device ${ }^{14)}$. The dynamic thickness (thickness during sliding) $D$ and real contact area $A$ were directly measured using multiple beam interferometry $(\mathrm{MBI})^{15}$ ). The friction force was normalized by the contact area and the shear stress $S$ (= $F / A)$ was evaluated. The schematic illustration of the

\footnotetext{
${ }^{a}$ Prior to the PDMS experiment, the influence of hexane on the structure and properties of the DDAB monolayer was examined. The droplets of blank hexane solution were spread on the DDAB surfaces and left for complete solvent evaporation, and then SFA friction measurements were made for the two DDAB monolayers in adhesive contact. The result for the DDAB film before the hexane treatment is shown in Figure 3. The friction force (shear stress) of the DDAB film before and after the hexane treatment was not different within the experimental error range $( \pm 10 \%)$, and the friction trace pattern was basically the same before and after the treatment. Further, AFM analysis did not show any structural changes of the DDAB surfaces by the treatment.
} 
sliding interface is shown in Figure 2. All the results reported in this paper were obtained with molecularly smooth hydrophobic substrates (wearless friction). The experimental room was kept at a fixed temperature of $23^{\circ} \mathrm{C} \pm 0.2^{\circ} \mathrm{C}$.

\section{Results and Discussion}

The dynamic thicknesses of the confined PDMS melt were $1.4 \mathrm{~nm}$ for the compressed system and $2.0 \mathrm{~nm}$ for the cast system (Table 1). Comparison with the width of the polymer backbone implies that the PDMS molecules in the both systems lay parallel to substrate surfaces; the dynamic thicknesses correspond to two molecular layer thick for the compressed system and three molecular layer thick for the cast system. For the compressed system, the dynamic thickness equaled the static hard-wall thickness (thickness before sliding). For the cast system, the static thicknesses did not always equal the dynamic thickness, larger thickness $(D=2.8$ $\mathrm{nm})$ was obtained at some contact positions. However, the thickness decreased to $2.0 \mathrm{~nm}$ when sliding was applied. We should note that all the static hard-wall thicknesses and the dynamic thicknesses for the two PDMS systems obtained here roughly equaled the integral multiples of the width of the PDMS backbone.

The dependence of the shear stress on sliding velocity for the PDMS systems is shown in Figure 3. The shear stress for the two DDAB monolayers (hydrophobic substrates) in adhesive contact $(P=0$ $\mathrm{MPa}$ ) is also shown for comparison. The shear stress for the cast system was larger than that of the compressed system, despite the large dynamic thickness. Generally, large dynamic thicknesses give low friction for most of the confined fluids; the result obtained here is opposed to the common behaviors.

Table 1 Results of the friction measurements for the two PDMS systems.

\begin{tabular}{|c|c|c|c|}
\hline & $\begin{array}{l}\text { Applied } \\
\text { pressure, } P \\
{[\mathrm{MPa}]}\end{array}$ & $\begin{array}{l}\text { Dynamic } \\
\text { thickness, } \\
D[\mathrm{~nm}]\end{array}$ & $\alpha$ \\
\hline $\begin{array}{c}\text { Compressed } \\
\text { system }\end{array}$ & 3.0 & 1.4 & $0.18 \pm 0.01$ \\
\hline Cast system & $\begin{array}{c}\text { 0 (adhesive } \\
\text { contact) }\end{array}$ & 2.0 & $0.18 \pm 0.02$ \\
\hline
\end{tabular}

When we discuss the effect of applied pressure on shear stress in Figure 3, the following consideration should be made. Although the applied pressure was zero for the cast system, self-adhesion produces attractive pressure at the contact interface. A simple estimation of the adhesion strength using the Laplace pressure equation gives $\sim 2.5 \mathrm{MPa}$ for the cast system. Therefore, the adhesive pressure was not very different between the two PDMS systems. This analysis is also inconsistent with the large shear stress of the cast system. ${ }^{b}$

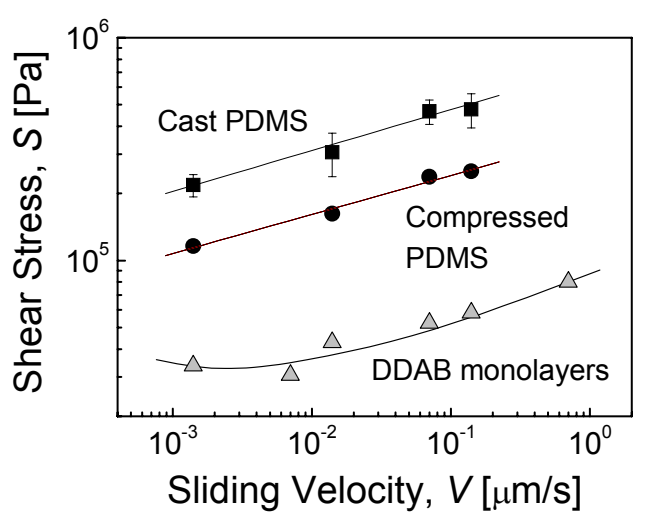

Fig.3 Shear stresses as a function of sliding velocity for the two PDMS systems. The shear stress for the DDAB/DDAB interface is also shown for comparison. Sliding conditions: $D=2.0 \mathrm{~nm}$ and $P=0 \mathrm{MPa}$ (adhesive contact) for the cast PDMS, $D=1.4 \mathrm{~nm}$ and $P=3.0 \mathrm{MPa}$ for the compressed PDMS, and $P=0 \mathrm{MPa}$ (adhesive contact) for the DDAB monolayers.

In Figure 3, we did not add error bars for the results of the compressed system, because it was difficult to keep applied load and pressure the same at different contact positions. The typical reproducibility of the friction measurements for the system at different contact positions was within the range of $\pm 15 \%$.

The relationship between shear stress $S$ and sliding velocity $V$ can be fit by the following equation:

$$
S \propto V^{\alpha}
$$

where $\alpha$ is a constant. The $\alpha$ values for the two PDMS systems are also included in Table 1 . The $\alpha$ parameter indicates the shear mechanisms whether "solidlike" friction or "liquidlike" rheological flow are observed: for ideal friciton $\alpha=0$ and for Newtonian flow $\alpha=$ $1^{4,6,16)}$. The $\alpha$ for the two PDMS systems are the same and rather close to the ideal value of solidlike friction. Therefore, the microscopic shear mechanisms of the two films are not very different and mainly governed by the slipping between molecules.

Figure 4 shows the possible confined structures of the two PDMS films. According to the results described above and in our previous paper ${ }^{8)}$, we estimate that the compressed PDMS system has a highly-ordered layer

\footnotetext{
${ }^{\mathrm{b}}$ For both systems, we could not perform the friction measurements under large applied pressure at different sliding velocities, because the contact interfaces damaged easily during sliding (the DDAB layers came off from mica surfaces and formed wear particles).
} 
structure (Figure 4a). The small hard-wall thickness (two molecular layers) suggests that molecules in the film are easily expelled from contact interface during squeezing, which implies the low interdigitation of molecules between layers. In addition, low friction also fits the expectation of the high ordering because of the easy slippage between ordered molecular planes ${ }^{8,9)}$. In contrast, the relatively large dynamic thickness of the cast system suggests that the molecules are less expellable than the compressed system, probably due to the molecular entanglement effect. Large friction for the cast system compared with the compressed system implies the interdigitation between molecules and molecular bridges between two sliding surfaces (Figure $4 \mathrm{~b})^{17,18)}$. If lateral sliding motions play a major role to enhance the molecular ordering, the cast film (three layer thick) should have transited into two layers during shear. However, the layering transition did not occur but surface damage occurred instead. This analysis leads to a conclusion that not the lateral sliding motions but the normal compression (squeeze out) is important to enhance the molecular ordering in confined PDMS films. a) Compressed System

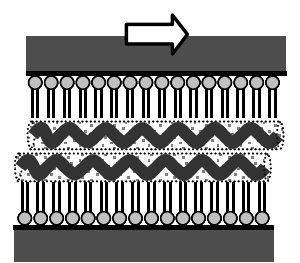

b) Cast System

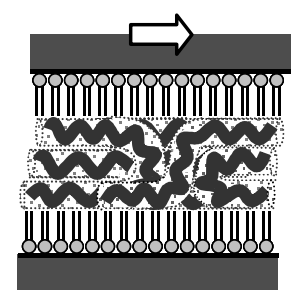

Fig.4 Schematic illustrations of the possible confined structures in the two PDMS systems. The molecules in the both films lay parallel to substrate surfaces, but the molecular conformations may be different. a) The molecules in the compressed system have rather a flat conformation and molecules belong to a specific layer. b) The molecules in the cast system could interdigitate between each other and molecular bridges may be formed between the two opposed surfaces.

Now we discuss how the film preparation procedures make the difference of the film structures. If the estimated models shown in Figure 4 are adequate, the major factor that determines the extent of ordering is the compression (squeeze out) of the PDMS molecules at large separations (large thicknesses). We speculate that the pressure-driven squeeze flow of molecules, which has a large shear rate, contributes to the flat molecular conformation and layering for the compressed system. Because of the large flexibility of siloxane backbone, the PDMS molecules at surfaces may be disentangled and flattened along with the squeeze flow (under a large shear rate). Recent X-ray scattering experiment supports this mechanism, shear-induced layering is observed for a PDMS melt at a liquid/solid interface ${ }^{19}$. In addition, boundary slip may occur at the hydrophobic DDAB surfaces ${ }^{20}$ ), which could also contribute to the layering. When the thickness of the PDMS melt reaches the hard-wall state by compression, the extent of the molecular layering on substrate should be already exceptionally high.

The squeeze out of molecules is also expected for the cast system when the two cast films are in adhesive contact. The thickness gradually decreases with time and reaches the final thickness by squeezing. However, the effect is not large enough for the long PDMS chains to order into layers. The molecular conformation in the system should be mainly governed during the casting of the nanometer thick film on substrate from solution. The random coil conformation in the solution becomes thinner during the solvent evaporation, and molecular chains lay parallel to surfaces due to the interaction between molecules and surfaces ${ }^{21)}$. However, there is no driving force for the molecules to disentangle the PDMS chains with each other; the extent of the ordering is low. This is consistent with the recent synchrotron X-ray reflectivity study of PDMS melts on surface ${ }^{22)}$. When the two cast PDMS films are made in contact, the molecules at interface interdigitate each other. Especially, the chain ends could have a large effect on the interpenetration and restructuring the surface segments ${ }^{23,24)}$. Contact pressure squeezes some amount of molecules as was mentioned, and the final film has an interdigitated (entangled) structure. Restructuring may induce molecular bridges between the two surfaces, which is presumably the major cause of high friction. Lateral sliding motions cannot rearrange the interdigitated film structures into well-ordered layers but induce surface damage.

In summary, we have investigated how long PDMS chains form layer structures in confinement using the SFA. The results imply that the pressure-driven squeeze flow of PDMS molecules at large thickness is important to form a well-ordered layer structure, which gives us a new insight to manipulate large molecules under confinement.

\section{Acknowledgements}

The author is grateful to Dr. Kazuo Maki for enlightening discussions, and to Kao Corporation for permission to publish this paper.

\section{References}

[1] Israelachvili, J., McGuggan, P. M. and Homola, A. M., "Dynamic Properties of Molecularly Thin Liquid Films," Science, 240, 4849, 1988, 189-191. 
[2] Granick, S., "Motions and Relaxations of Confined Liquids," Science, 253, 5026, 1991, 1374-1379.

[3] Bhushan, B., Israelachvili, J. and Landman, U., "Nanotribology: Friction, Wear and Lubrication at the Atomic Scale," Nature, 374, 6523, 1995 , 607-616.

[4] Israelachvili, J. and Berman, A. D., Surface Forces and Microrheology of molecularly thin liquid films, CRC Handbook of Micro/Nanotribology, 2nd ed., ed. Bhushan, B., CRC Press, Boca Raton, FL, 1999, 371.

[5] Cai, L., Peanasky, J. and Granick, S., "Nanorheology of Polymers," Trends Polym. Sci., 4, 2, 1996, 47-51.

[6] Yamada, S., Nakamura, G. and Amiya, T., "Shear Properties for Thin Films of Star and Linear Polymer Melts," Langmuir, 17, 5, 2001, 1693-1699.

[7] Israelachvili, J. N., Intermolecular and Surface Forces, 2nd ed., Academic Press, London, 1991, 288-311.

[8] Yamada, S., "Layering Transitions and Tribology of Molecularly Thin Films of Poly(dimethylsiloxane)," Langmuir, 19, 18, 2003, 7399-7405.

[9] Granick, S., Lin, Z. and Bae, S. C., "Molecules Squeezed and Stroked," Nature, 425, 6957, 2003, 467-468.

[10] Fetters, L. J., Lohse, D. J., Milner, S. T. and Graessley, W. W., "Packing Length Influence in Linear Polymer Melts on the Entanglement, Critical, and Reptation Molecular Weights," Macromolecules, 32, 20, 1999, 6847-6851.

[11] Horn, R. G. and Israelachvili, J. N., "Molecular Organization and Viscosity of a Thin Film of Molten Polymer between Two Surfaces as Probed by Force Measurements," Macromolecules, 21, 9, 1988, 2836-2841.

[12] Gee, M. L. and Israelachvili, J. N., "Interactions of Surfactant Monolayers across Hydrocarbon Liquids,” J. Chem. Soc. Far. Trans., 86, 24, 1990, 4049-4058.

[13] Israelachvili, J. and McGuiggan, P. M., "Adhesion and Short-Range Forces Between Surfaces I New Apparatus for Surface Force Measurements," J. Mater. Res., 5, 10, 1990, 2223-2231.
[14] Luengo, G., Schmitt, F. J., Hill, R. and Israelachvili, J. N., "Thin Film Rheology and Tribology of Confined Polymer Melts: Contrasts with Bulk Properties," Macromolecules, 30, 8, 1997, 2482-2494.

[15] Israelachvili, J., "Thin Film Studies Using Multiple Beam Interferometry," J. Colloid Interface Sci., 44, 2, 1973, 259-272.

[16] Yamada, S., "General Shear Thinning Dynamics of Confined Fluids," Tribol. Lett., 13, 3, 2002, 167-171.

[17] Raviv, U., Tadmor, R. and Klein, J., "Shear and Frictional Interactions between Adsorbed Polymer Layers in a Good Solvent," J. Phys. Chem. B, 105, 34, 2001, 8125-8134.

[18] Tadmor, R., Chen, N. and Israelachvili, J., "Normal and Shear Forces between Mica and Model Membrane Surfaces with Adsorbed Hyaluronan," Macromolecules, 36, 25, 2003, 9519-9526.

[19] Yu, C., Evmenenko, E., Kmetko, J. and Dutta, P., "Effects of Shear Flow on Interfacial Ordering in Liquids: X-ray Scattering Studies," Langmuir, 19, 23, 2003, 9558-9561.

[20] Schmatko, T., Hervet, H. and Leger, L., "Friction and Slip at Simple Fluid-Solid Interfaces: The Roles of the Molecular Shape and the Solid-Liquid Interaction," Phys. Rev. Lett., 94, 24, 2005, 244501.

[21] Evmenenko, G., Dugan, S. W., Kmetko, J. and Dutta, P., "Molecular Ordering in Thin Liquid Films of Polydimethylsiloxanes," Langmuir, 17, 13, 2001, 4021-4024.

[22] Evmenenko, G., Mo, H., Kewalramani, S. and Dutta, P., "Conformational Rearrangements in Interfacial Region of Polydimethylsiloxane Melt Films," Polymer, 47, 3, 2006, 878-882.

[23] Maeda, N., Chen, N., Tirrell, M. and Israelachvili, J., "Adhesion and Friction Mechanisms of Polymer-on-Polymer Surfaces," Science, 297, 5580, 2002, 379-382.

[24] Chen, N., Maeda, N., Tirrell, M. and Israelachvili, J., "Adhesion and Friction of Polymer Surfaces: The Effect of Chain Ends," Macromolecules, 38, 8, 2005, 3491-3503. 